\section{En busca de una tradición perdida}

Linazasoro y su libro sobre las ciudades vascas

\section{In search of a lost tradition}

Linazasoro and his book on basque cities
Resumen. El libro sobre las ciudades vascas de José Ignacio Linazasoro fue concebido en un clima cultural de fuerte presen-cia del discurso morfo-tipológico proveniente de Italia; sin embargo, en él su autor no se limitó a aplicar una teoría general a un caso particular. Además de profundizar en el conocimiento de la evolución urbana y territorial de un conjunto de núcleos de fundación medieval sobre los que en gran medida se asienta el sistema de ciudades del País Vasco, Linazasoro puso con él las bases de determinados aspectos que desarrollaría en su trayectoria poste-rior como arquitecto; en particular, el trabajo con las preexistencias, la atención al modo en que se relacionan lo antiguo con lo nuevo y el interés por las cuestiones matéricas. En este artículo se presta particular atención, de una parte, a las circunstancias culturales y profesionales en que surgió el libro y, de otra, a su significado para la posterior obra construida de Linazasoro.
Victoriano Sainz Gutiérrez

\section{Palabras Clave}

José Ignacio Linazasoro

Ciudades vascas

Morfología urbana

Tipología edificatoria

Permanencias
ABSTRACT. The book on the Basque cities by José Ignacio Linazasoro was concetred in a cultural climate with a strong pres-ence of the Italian himself to applying a gen-eral theory to a particular case. In addition to deepening the knowledge of the urban and territorial evolution of a
group of towns of medieval foundation on which the system of cities of the Basque Country is largely based, Lina-zasoro laid with him the bas special-ly, the wo (n) in which the book arose and, on the other, on its significance for the later Basque cities wri of Linazasoro. KEY WORDS. Jose Ignacio Lin 
El impacto de las ideas de la Tendenza en la cultura arquitectónica española estuvo ligado, en un primer momento al menos, a la difusión de sus escritos, pues es sabido que quienes estaban en el origen de ese movimiento, nacido en Italia a mediados de los años sesenta, dieron una particular importancia al discurso teórico. Era un rasgo que compartían con los restantes arquitectos italianos de su generación, caracterizados por "considerar la crítica y la historia como instrumentos de proyectación, utilizar directamente el razonamiento teórico como razonamiento de proyecto y pensar en la arquitectura como conocimiento, rehusando separar teoría y realidad" ${ }^{1}$.

En el caso de la Tendenza, esa reflexión teórica giró en gran medida alrededor de un análisis arquitectónico de la ciudad, planteado mediante el estudio de las relaciones entre la morfología urbana y la tipología edificatoria. Tras las huellas de Saverio Muratori, este método de análisis se fue concretando durante los tres años en que Carlo Aymonino y Aldo Rossi compartieron en Venecia la docencia de lo que más tarde se llamaría Composición Arquitectónica. Junto a los volúmenes que recogían las clases impartidas entre 1963 y 1966, publicaron además sendos libros que tuvieron un carácter casi fundacional para el movimiento: Origini e sviluppo della città moderna (1965), de Aymonino, y L'architettura della città (1966), de Rossi.

La puerta de entrada en nuestro país para esas nuevas ideas sería Barcelona y, de hecho, su Escuela de Arquitectura no tardó en comenzar a difundirlas a través del Laboratorio de Urbanismo, fundado por Manuel de Solà-Morales en 1968. De otra parte, ya el año anterior el propio Rossi había participado en el Pequeño Congreso celebrado en Tarragona, donde dio la conferencia inaugural, invitado por un grupo de arquitectos catalanes capitaneados por Oriol Bohigas; allí lo conoció personalmente Rafael Moneo, que poco después obtendría una cátedra en Barcelona. En este contexto, no tardarían en aparecer, publicadas por la editorial Gustavo Gili, las versiones castellanas de los mencionados libros, comenzando por el de Rossi, traducido por Salvador Tarragó, que fue puesto a la venta en la primavera de 1971.

$\mathrm{Al}$ calor de aquel clima cultural se gestó la que fue una de las primeras monografías redactadas en nuestro país con el enfoque morfo-tipológico difundido por la Tendenza; se trata del estudio sobre las ciudades vascas de José Ignacio Linazasoro, que vio la luz en 1978 bajo el título Permanencias y arquitectura urbana (figura 1). Si se propone revisitarlo ahora, no es sólo por su carácter pionero, sino también y sobre todo porque puede servir para ilustrar el modo en que ese enfoque fue recibido entre los arquitectos españoles: "No queríamos asumir, sin más, el ambiente intelectual italiano -ha dicho recientemente Linazasoro-: queríamos extrapolarlo, traducirlo a nuestro contexto. Esto pasaba por reivindicar lo local [...]; intentábamos resucitar una tradición perdida" ${ }^{2}$.

\section{Los contactos de Linazasoro con la cultura italiana}

Cuando a comienzos de los años setenta Linazasoro dejó Pamplona y se trasladó a Barcelona para acabar sus estudios de Arquitectura, el análisis urbano de los italianos era, pues, ya suficientemente conocido por los profesores que se ocupaban de impartir las materias urbanísticas en la Escuela de la capital catalana. Sin embargo, no serían éstos, sino Moneo quien le animara a leer La arquitectura de la ciudad, entonces recién publicado en español y que despertó en él un notable interés por conocer mejor aquel filón de ideas que allí se encontraban sólo enunciadas: "El libro de Rossi fue un puente para otras lecturas como La città di Padova, a través de la que descubrí la técnica del rilievo tipológico que ya aplicaría a algunos trabajos del último curso de la Escuela de Barcelona o a mi proyecto fin de carrera" ${ }^{3}$

Éste consistió en proyectar unas viviendas en el centro histórico de Pamplona y, para afrontarlo, Linazasoro trató de servirse de los estudios sobre la tipología de los italianos, "levantando alzados urbanos e intentando incardinar el proyecto en la memoria del lugar" ". Ahora bien, si Rossi resultó ser un descubrimiento fundamental para el arquitecto donostiarra fue porque, más allá de ser la moda cultural del momento, le ofrecía un modo concreto de vincular la historia y el dibujo a través del proyecto, pues el interés por la arquitectura histórica, singularmente la románica, se encontraba -según él mismo ha relatado ${ }^{5}$ - en el origen de su decisión de hacerse arquitecto. Ciertamente, el buen resultado académico obtenido con aquel proyecto fin de carrera debió animarlo a seguir por ese camino.

Linazasoro regresó a San Sebastián en 1972, donde daría comienzo su vida profesional. Allí, en unos años en que escaseaban los encargos como consecuencia de la crisis económica iniciada en 1973, compatibilizó el ejercicio de la arquitectura con una intensa actividad cultural en el seno de la delegación guipuzcoana del Colegio de Arquitectos Vasco-Navarro. Junto a Miguel Garay, con quien también colaboró profesionalmente en esos años,

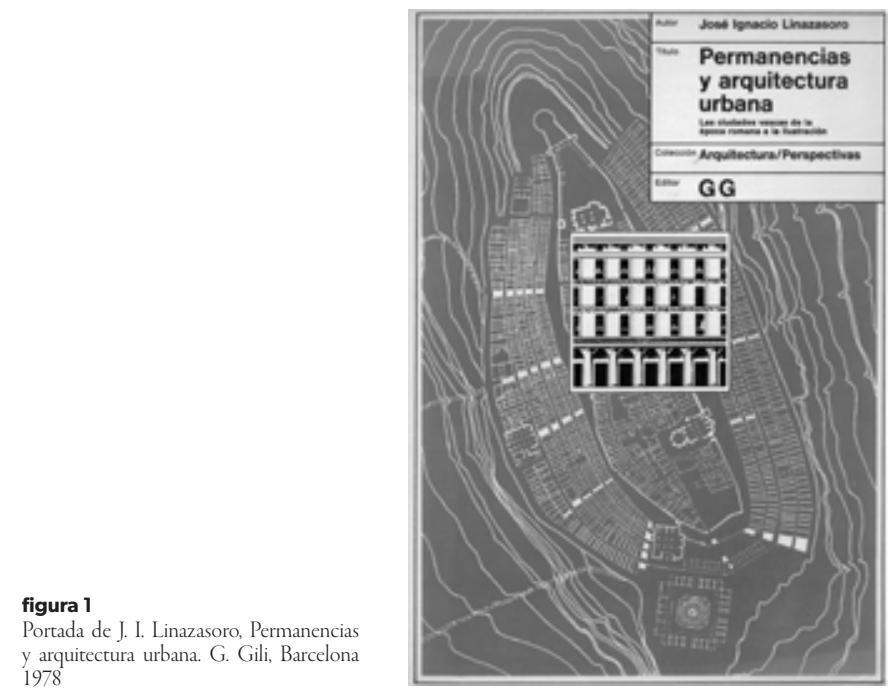
y arquitectura urbana. G. Gili, Barcelona
1988

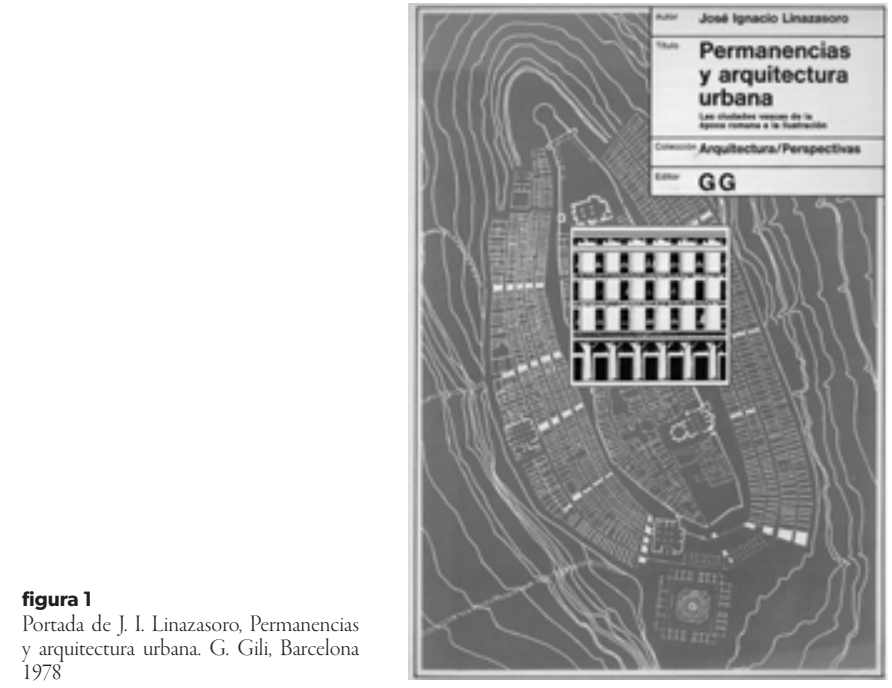


se encargó de la comisión de cultura del Colegio y ambos organizaron las llamadas Semanas de Arquitectura, por las que pasaron muchos de los protagonistas de los más vivos debates del momento; entre ellos, claro está, los italianos. Aymonino, Rossi, Grassi, Tafuri o Scolari estuvieron en San Sebastián para dar conferencias o participar en cursos, lo cual permitió a Linazasoro tener un conocimiento de primera mano de sus puntos de vista y establecer una relación más o menos personal con ellos.

Fueron unos años en los que los Colegios de Arquitectos españoles, a través de las reuniones de sus archivos históricos, mantuvieron frecuentes y estrechos contactos. Esas reuniones, impulsadas por Tarragó desde Barcelona, permitieron a Linazasoro conocer a Aymonino en Valencia y a Rossi en Santiago de Compostela, en marzo y diciembre de 1973, respectivamente. De resultas de todo ello, ese mismo año Aymonino viajó a San Sebastián para dar una conferencia sobre la actuación residencial del Gallaratese; al año siguiente fue Rossi quien visitó la capital guipuzcoana para dar dos conferencias, una sobre los problemas de los centros históricos y otra sobre su propia arquitectura; y, finalmente, en 1977 también Grassi estuvo en San Sebastián como invitado al curso para posgraduados, organizado el Colegio de Arquitectos.

Durante esta última visita, Grassi manifestó a Linazasoro su interés por publicar en Gustavo Gili una selección de sus escritos sobre arquitectura, donde finalmente aparecerían unos años más tarde con un prólogo del arquitecto donostiarra. En ese texto encontramos lo que por entonces era la posición de Linazasoro en relación con la arquitectura, en gran medida influenciada por las ideas de la Tendenza: "A lo largo de esta introducción -escribía- he querido tratar los temas y problemas que preocupan a Giorgio Grassi y a algunos arquitectos entre los que me incluyo" ${ }^{6}$. Y entre esas cuestiones se hallaba la afirmación de que la identidad de la arquitectura como disciplina sólo podía hallarse en la historia, que es donde cabía reconocer su contenido más específico, según la célebre afirmación grassiana: "La arquitectura son las arquitecturas, no existe una teoría de la arquitectura que no sea a la vez una experiencia de la arquitectura" 7 .

Y esa experiencia se da siempre en la ciudad, en la realidad concreta de los "hechos urbanos", en el sentido que diera Rossi a esta expresión. De ahí que una de las primeras consecuencias que el contacto con el universo conceptual de la Tendenza tuvo para Linazasoro fuese un particular empeño por conocer mejor las villas de fundación medieval de su tierra natal, tanto en su génesis como en su evolución posterior (figura 2). Seguía en esto la recomendación del arquitecto milanés, que había escrito: "En los estudios urbanos nunca daremos bastante importancia al trabajo monográfico, al conocimiento de los hechos urbanos particulares. Omitiendo éstos -advertía-, aun en los aspectos de la realidad más individuales, particulares e irregulares, pero por ello también más interesantes, terminaremos por construir teorías tan artificiales como inútiles" ${ }^{8}$.

No se trataba, pues, de aplicar sin más un método considerado más o menos universalmente válido a un caso particular, sino de profundizar en una realidad urbana concreta, con su propia especificidad. Así lo ha señalado, por ejemplo, quien fuera su asistente en el Politécnico de Zúrich en la primera mitad de los años setenta: "En lugar de iniciarnos en la cultura lombarda e italiana, [Rossi] se esforzó por desarrollar en nosotros el interés por la tradiciones arquitectónicas y culturales suizas. De este modo los estudiantes comenzaron a estudiar las estructuras de las ciudades y los pueblos suizos, y a investigar sus vínculos con las diferentes tradiciones constructivas" ${ }^{9}$. Y eso fue exactamente lo que hizo Linazasoro con las ciudades vascas.

\section{Linazasoro y Rossi ante un tema común de estudio}

Es por eso que el trabajo de Linazasoro no debe ser leído sólo en relación con el descubrimiento del análisis urbano impulsado por la Tendenza, que fue característico de los primeros años setenta ${ }^{10}$, sino que necesita ser inscrito también dentro de un clima de particular interés por el desarrollo urbano en el País Vasco, compartido por los arquitectos que, desde finales de los años sesenta, se reunían en torno a la figura de Luis Peña Ganchegui en su estudio de San Sebastián. Fruto de ese interés fue sin duda la invitación a participar en la III Semana de Antropología Vasca, celebrada en Bilbao en abril de 1973, donde Peña e Iñaki Galarraga disertaron sobre las peculiaridades urbanas de las ciudades vascas ${ }^{11}$.

La conferencia inaugural de esa Semana había corrido a cargo de Julio Caro Baroja, quien en junio de ese mismo año daría en San Sebastián, invitado por el Colegio de Arquitectos, la conferencia titulada: "La arquitectura y el urbanismo en el concepto de pueblo vasco". Era un asunto del que el antropólogo e historiador se había ocupado de manera recurrente a lo largo de su trayectoria investigadora y sobre el que ayudaría a profundizar a un grupo de jóvenes arquitectos interesados en el tema, a través de las numerosas

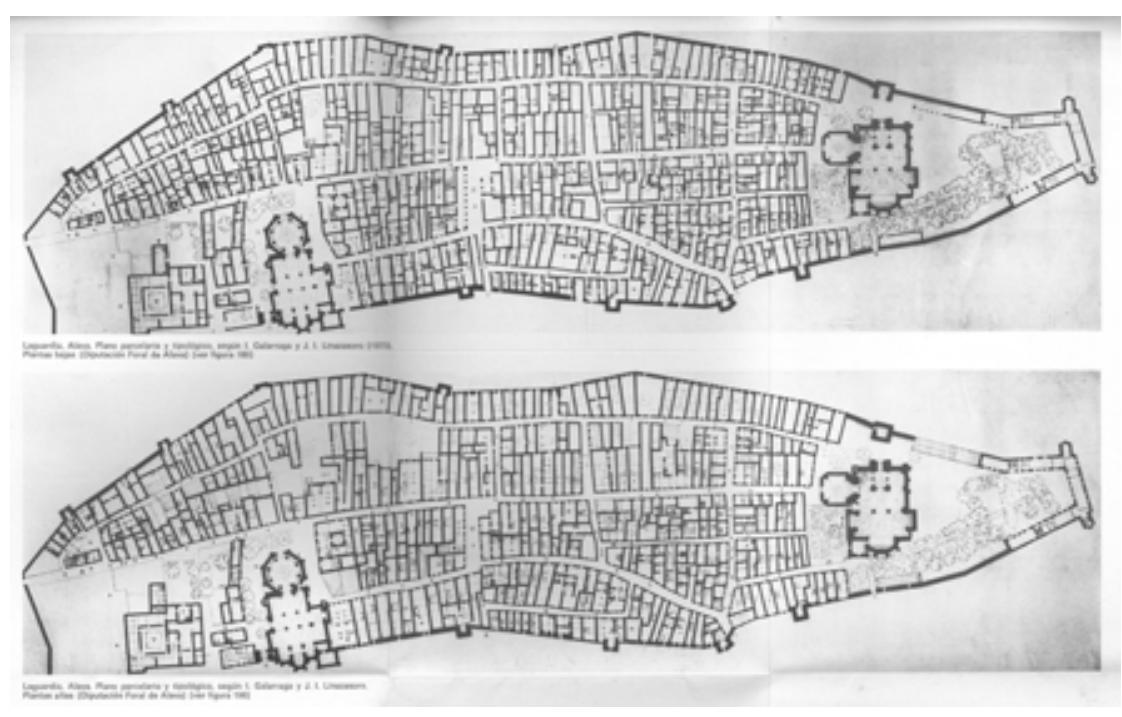


explicaciones dadas sobre el terreno en una serie de visitas organizadas por la comisión de cultura del Colegio en los meses sucesivos. Se constituyó así en referente intelectual llamado a convertirse en la figura de reconocido prestigio capaz de dar un soporte historiográfico y teórico a aquella aventura (figura 3), uno de cuyos frutos sería el libro de Linazasoro.

"A base de planos viejos -escribió luego Caro Baroja- les fui explicando lo que sabía acerca de aquellas poblaciones creadas en fechas medievales, por razones varias. La documentación gráfica era, a todas luces, insuficiente, y varios de los excursionistas demostraron al final la voluntad de superarla" ${ }^{12}$. De hecho, Linazasoro procedió a hacer los levantamientos de esas villas, que sirvieron de base para el análisis tipológico que Galarraga y él hicieron sobre la base de las fuentes documentales que pudieron localizar. Cuando se encontraban realizando este trabajo, tuvo lugar la visita de Rossi a San Sebastián y, durante los días que pasó en el País Vasco, lo acompañaron a ver algunas de esas poblaciones, que le interesaron particularmente porque, aunque sus anfitriones no tuvieran noticia de ello, él mismo se hallaba ocupado en el estudio de las poblaciones rurales del cantón suizo del Tesino ${ }^{13}$ (figura 4).

La redacción, en paralelo y de manera independiente, del libro de Linazasoro sobre las ciudades vascas y del de Rossi sobre las tesinesas presentaba una evidente afinidad de planteamientos. Si se analiza cómo procedieron en sus respectivas investigaciones se observa que Paul Hofer fue para Rossi lo que José María Lacarra para Linazasoro, un gran conocedor de la historia urbana medieval al que acudir; como ambos tuvieron también su antropólogo de referencia: Jakob Hunziker, en el caso del arquitecto milanés y sus alumno suizos, y Caro Baroja, en el de los jóvenes arquitectos guipuzcoanos. Al igual que los dos estudios llevan a cabo un análisis de las diferentes tipologías edificatorias y sobre todo de su inserción en la forma urbana, y de la relación de ésta con el territorio; fue este un aspecto que interesó particularmente a Rossi cuando conoció, de la mano de Linazasoro y Garay, las ciudades vascas de fundación medieval.

En su libro, Linazasoro denominará "ciudades-camino" a las primeras villas fundadas a comienzos del siglo XI (figura 5), caracterizadas precisamente por "el 'uso' del camino como material físico de construcción de la ciudad. [...] La calle medieval, en cuanto estructura básicamente urbana, todavía no se diferenciara del camino como estructura básica de comunicación" ${ }^{14}$. Es cierto que la estructura urbana no tardaría en adquirir una mayor complejidad, mediante la construcción de la muralla que delimitaba un perímetro, en el interior del cual se procedió a lotear las manzanas resultantes del trazado de calles paralelas a la principal, pero la forma primigenia asociada al camino seguirá siendo reconocible incluso hasta nuestros días; por eso pudo anotar Rossi: "Camino y calle mayor no cambian de sección y se prolongan el uno en la otra. Desde la muralla se ve perderse la calle mayor en las colinas como carretera" ${ }^{15}$.

Era, pues, común a ambos esa visión territorial de lo urbano entendido como manufactura, como hecho construido (figuras 6,7 ). El arquitecto milanés ya la había explorado en su estudio de finales de los años sesenta sobre las ciudades vénetas ${ }^{16}$, pero al enfrentarse a las ciudades del Tesino pronto comprendió que la aproximación debía ser diversa, pues el entorno rural suizo también lo era. Ahora bien, como apuntara Luca Ortelli en su reseña a la edición italiana del libro sobre el territorio tesinés, Rossi era ajeno a cualquier visión romántica de lo vernáculo, de ahí que frente a "la consideración abstracta y gratuita de los consabidos 'cuadros paisajistas', propuestos con frecuencia en Suiza para su protección, aquí se sustituye por la idea, rica en desarrollos e implicaciones operativas, del territorio como ciudad" ${ }^{17}$. Y esa idea era compartida por Linazasoro.

Pero lo que más los aproximaba era que los dos tenían una visión proyectual del problema que estaban estudiando. Por eso estaban tan distantes de la retórica de la protección patrimonial más extendida, ya que eran particularmente

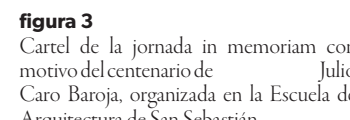
motivodel centenariode
Caro Baroja, organizada en la Escueda
Arquitectura de San Scebastián

gura 4 Levantamiento tipológico de Indemini en
dTesino (Suiza). realizado or A A Rosit

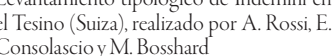

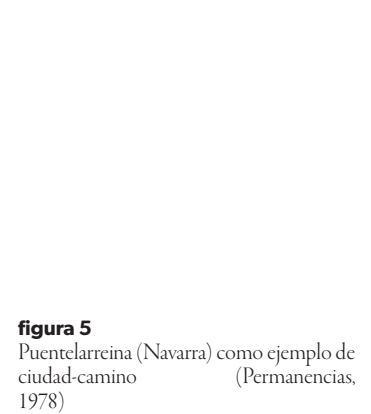

figura 6
Notas de

Notas de Aldo Rossi sobre su visita al
Pais Vasco en 1974, con esquema de alle porticada (Quaderni azzuriti n. maggio 1974)

\section{JULIOCAROBAROJA}

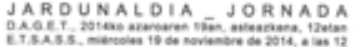

HIZLARIAK _ INTERVINIENTES $=$
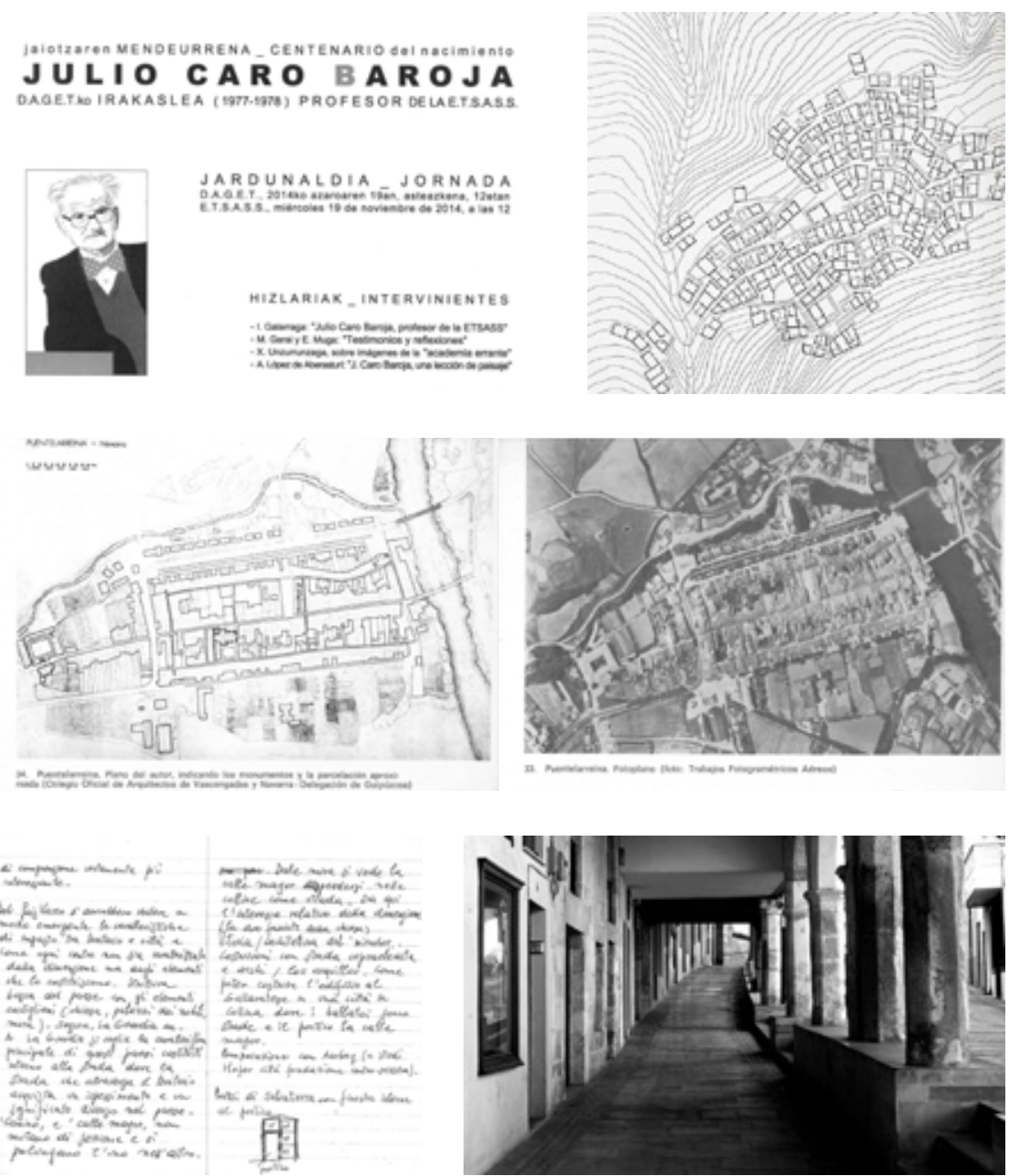

昷

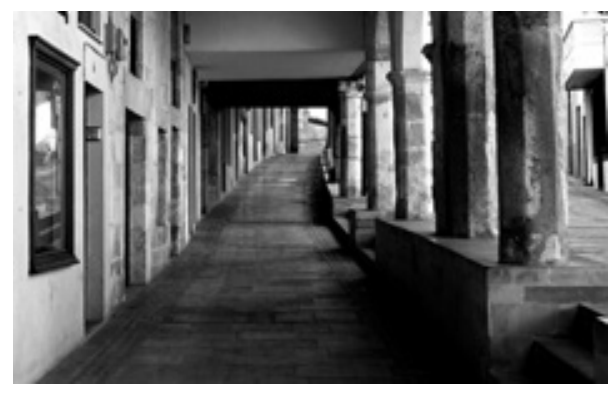


conscientes de lo que cabría denominar la "muerte" del patrimonio. En su libro sobre el territorio tesinés, Rossi escribió: "Parece casi imposible que la cualidad que la piedra ha asumido a lo largo del tiempo pueda ser de algún modo restaurada, en el sentido de una nueva funcionalidad del edificio. [...] Su destino se identifica con el significado histórico que es a la vez el significado actual: esto es, una vez más, el abandono" ${ }^{18}$. Su punto de vista es el del arquitecto que ve esa investigación como una "ocasión para trabajar en torno a la hipótesis de la ciudad análoga", y para ello "la descripción debe alcanzar el grado de la invención, más allá de las descripciones de los planos y los levantamientos" ${ }^{19}$.

Y algo semejante le sucedía a Linazasoro, quien a propósito de su libro sobre las ciudades vascas ha dicho: "Con aquel trabajo, el más analítico de los que he realizado, lo que me quedó claro es que no tenía vocación de investigador ni de erudito, sino tan sólo de explorador de arquitecturas que pudiesen generar procesos arquitectónicos, es decir, de proyecto" ${ }^{20}$. Y, de hecho, el libro se cierra con un apéndice donde el arquitecto donostiarra intenta explorar las posibilidades de aplicación de la teoría rossiana de la arquitectura análoga, como método compositivo, a los análisis morfo-tipológicos realizados para las ciudades estudiadas en él. En ese contexto el arquitecto donostiarra ensaya diversas operaciones de carácter lógico-formal, en forma de collage -como hiciera Rossi-, que le permiten "deducir conclusiones de orden compositivo difícilmente explicitables por otros medios” ${ }^{21}$ (figuras 8, 9).

\section{Significado del libro para la obra posterior de Linazasoro}

Probablemente la intuición más original de su libro sobre las ciudades vascas de fundación se encuentre en la tesis de que las "permanencias" en esos conjuntos urbanos por él analizados no habría que buscarlas acudiendo sólo a las tipologías edificatorias, como hacía la Tendenza, sino también a las técnicas constructivas. De ahí, por ejemplo, la insistencia de Linazasoro en el papel desempeñado por los muros medianeros de piedra de la casa medieval urbana convertidos en "elementos sustentantes de otro tipo de edificios, perdido ya su sentido divisorio" 22 (figura 10). O en la reutilización de los restos de torres góticas preexistentes para la construcción de las casonas solariegas renacentistas, evidenciando "la unión, en un mismo edificio, de técnicas y materiales diferentes, dando lugar a composiciones heterogéneas y, en este sentido, poco ortodoxas" ${ }^{23}$.

Y es que, más que la oportuna contribución al conocimiento del proceso de formación y transformación de las ciudades vascas, resultante de un encomiable esfuerzo por grafiarlo e interpretarlo, lo que parece más importante destacar de ese libro es el empeño de su autor, entonces joven arquitecto, por ahondar en las raíces urbanas de la arquitectura histórica, para encontrar ahí muchas de las claves de lo que sería su obra construida posterior. Pensemos en las viviendas de Mendigorría (1978-80), realizadas con Garay, donde quizá por primera vez aparece en la arquitectura de Linazasoro, junto al interés por subrayar la figura 8
Aldo Ros i, Collage sin título (1968)

figura 9

J. I. Linazasoro, Collage con la planta
de. Puentelarreina y la laza de Vitoria
(Permanencias, 1978)

figura 10 Muros antiguos en el interior de una
manzana de Puentelarreina (Permanencias,
1978)
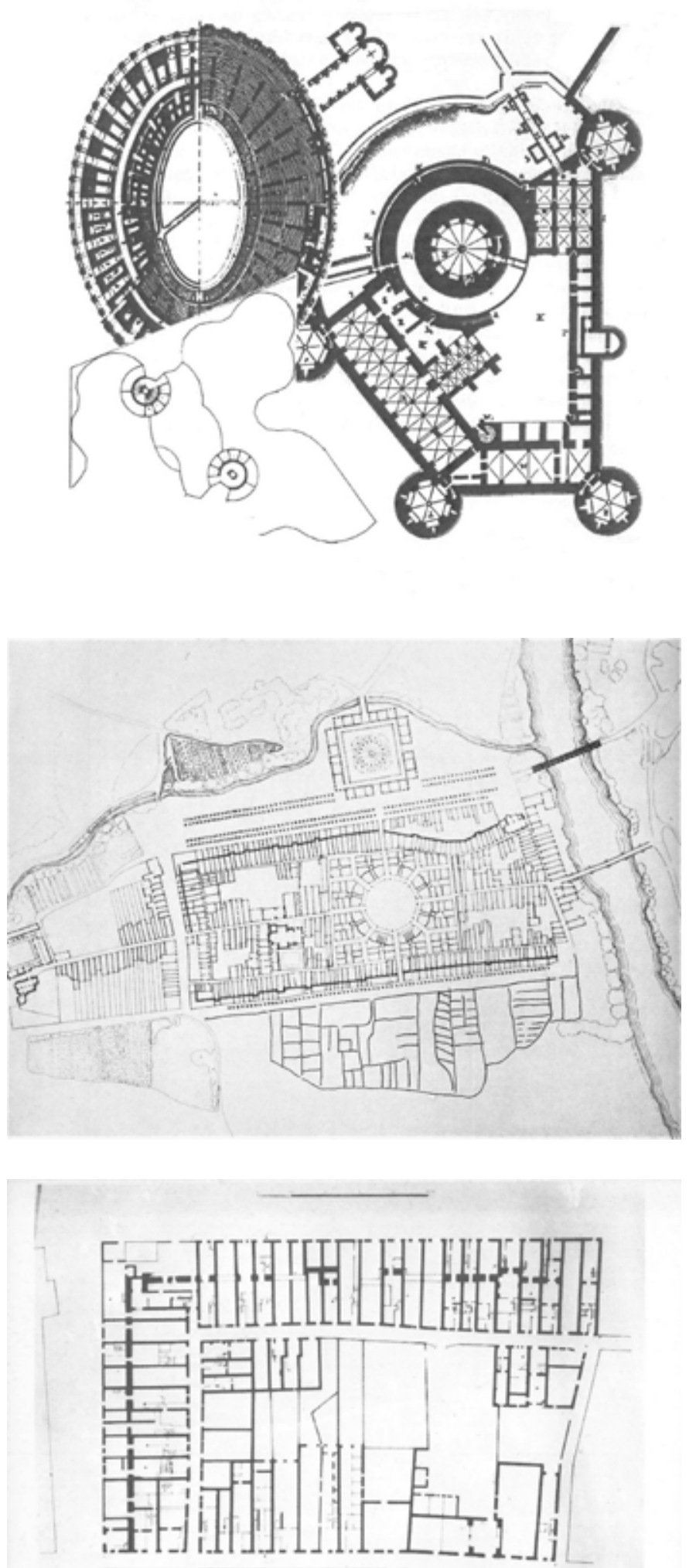

: 
urbanidad, la búsqueda de "un lenguaje más apoyado en la construcción y en los elementos e imágenes tradicionales, [...] una construcción -en palabras de Antón Capitel- que persigue lo texturado" 24 .

De ese proyecto ha dicho Linazasoro que lo considera, dentro de su trayectoria "el inicio de una nueva manera de entender la continuidad de la historia -la permanencia- desde la construcción, la materia y la tipología" ${ }^{25}$. Y ya que, en la posterior evolución de su obra, el trabajo con las preexistencias ha sido frecuente, se entiende que los aspectos matéricos y contextuales hayan ido adquiriendo una particular relevancia en su modo de proyectar. Es algo que se plantea por primera vez en el libro sobre las ciudades vascas, que también por eso puede ser considerado como el primer paso en una dirección que, andando el tiempo, nos ha legado algunas de las obras más emblemáticas del arquitecto donostiarra.

Es quizá en el proyecto para transformar las ruinas de las antiguas Escuelas Pías de San Fernando, situadas en el barrio madrileño de Lavapiés, en un centro cultural (1997-2004), donde Linazasoro se ha enfrentado con mayor grado de madurez al problema de cómo integrar los restos de un edificio preexistente en otro nuevo, que por eso mismo contiene una diversidad de estratos y da lugar a un conjunto complejo y heterodoxo, en cuanto que integra piezas o partes $\mathrm{de}$ muy distinto carácter, tanto desde el punto de vista de la materialidad como de la composición (figura 11). Era algo con lo que se hallaba familiarizado a través de su estudio del modo en que los palacios urbanos de las villas vascas se habían construido a partir de antecedentes medievales, y de lo que en cierta manera con este proyecto ofrece su particular versión desde una perspectiva contemporánea, optando por aquella clave constructiva crítica que ya había explorado en su intervención en la iglesia de Medina de Rioseco (1985-88)

En las Escuelas Pías madrileñas, además de reutilizar-comohicieran tantas casas señoriales vascas- numerosos elementos preexistentes para su construcción, Linazasoro lo ha hecho prestando particular atención a su integración en e conjunto durante el proceso constructivo; así lo ha testimoniado José María García del Monte, que colaboró con él en este proyecto, al referirse al modo en que fueron incorporando los nuevos descubrimientos hechos en obra en el entorno de la antigua iglesia en ruinas: "un sótano que dio lugar a bajar los aseos a un lugar sin duda mejor, una cripta que se empleó a nuestro favor para incrementar la tensión vertical en una última grieta generada en la pared de la altar, unas losas de piedra sorprendentes, una puerta con rejas en el altar que se reveló sobrevenida y se desmontó para integrar sus piedras en el muro de la fachada de la plaza, etc." ${ }^{26}$. Se trata de un empirismo que parece aprendido de aquellos maestros de obras que trabajaron siglos atrás en su tierra natal.

La intervención de Linazasoro en la plaza de la catedral de Reims (2003 08) constituye un proyecto que le ha permitido medirse con una cuestión cuyo punto de arranque se halla en el libro de las ciudades vascas; allí, al

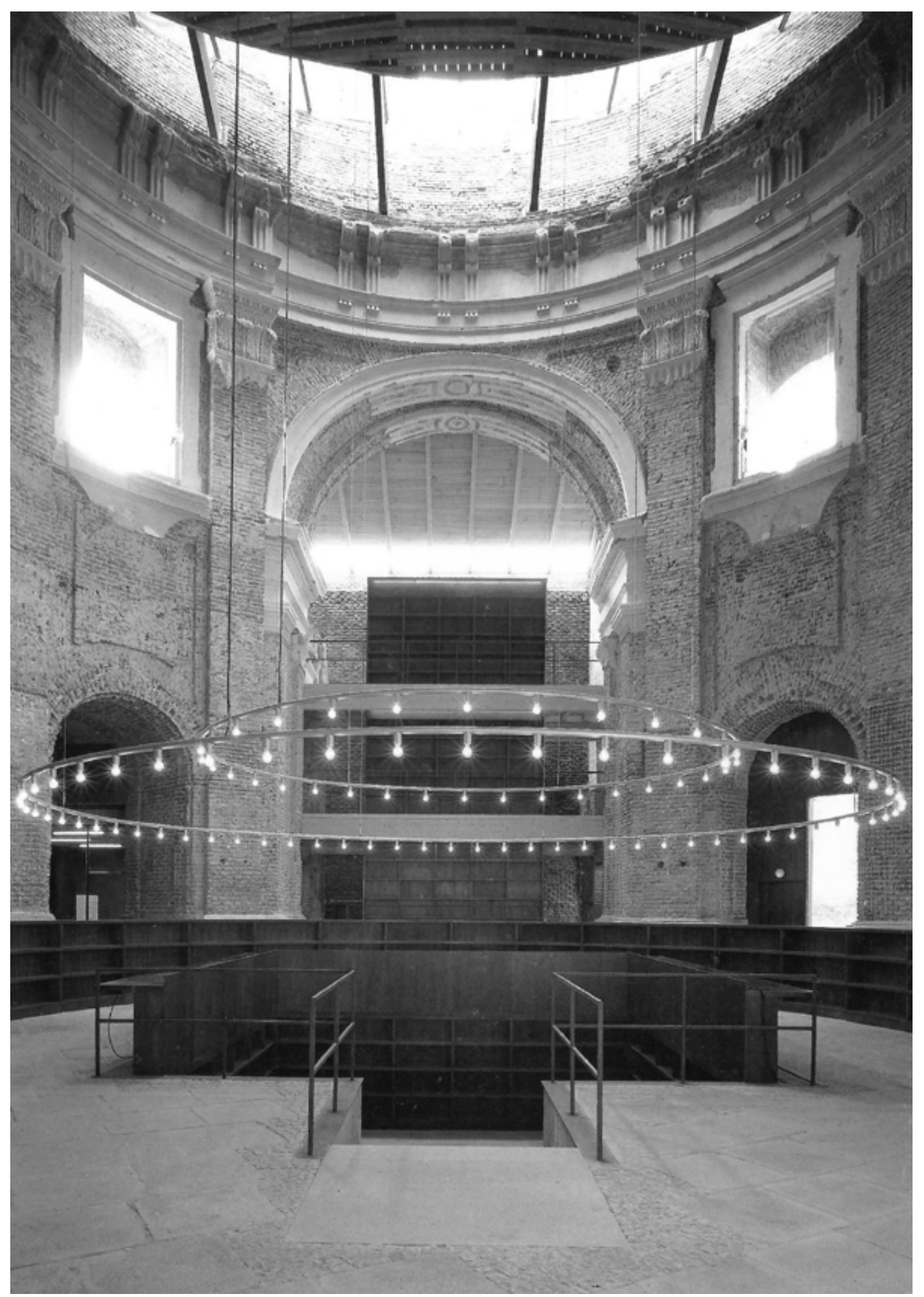


ocuparse de las permanencias monumentales y su relación con la forma de la ciudad, el arquitecto donostiarra evocaba la actual situación de muchas catedrales góticas, en particular las francesas, que se han visto desgajadas de las construcciones que las rodeaban y las insertaban en el tejido urbano, para convertirse en monumentos aislados ${ }^{27}$ (figura 12). Desde un punto de vista estrictamente proyectual, había estudiado cómo revertir esa situación en los ejercicios que planteó a sus estudiantes de San Sebastián y Valladolid a comienzos de los años ochenta ${ }^{28}$, pero sólo cuando ganó el concurso de 2002 para la reordenación del entorno de la catedral remense tuvo ocasión de enfrentarse en primera persona a ese problema, y lo hizo en un entorno particularmente emblemático, por tratarse de un lieu de mémoire ligado a la coronación de los reyes franceses.

En coherencia con lo que siempre había pensado, el proyecto de Reims intenta resituar la catedral en un entorno cuya escala respondiese a la de la ciudad anterior a los derribos decimonónicos (figura 13), produciendo "una versión más esencial, con una geometría más clara aunque siempre irregular para no competir con la geometría de la catedral" ${ }^{29}$. Para ello Linazasoro procede a construir una sutil topografía y a emplear diversos pavimentos le permiten evocar el antiguo tejido urbano medieval demolido, a través del juego entre lo mineral y lo vegetal, configurando un espacio público, unitario y articulado a la vez, donde son las diversas texturas las que inducen los posibles usos de cada ámbito, sin imponer nunca una solución única. La fragmentación del inhóspito vacío preexistente, llevada a cabo mediante la creación de unas suaves plataformas que sirven de soporte para los volúmenes definidos por la vegetación, le permite evocar el viejo tejido medieval: es quizá una aplicación sabia y actualizada, menos formalista, de aquel principio de la analogía que Linazasoro había tratado de definir en el capítulo final de su libro sobre las ciudades vascas.

Los proyectos mencionados permiten entender por qué Linazasoro ha podido afirmar, refiriéndose a Permanencias y arquitectura urbana: "Este ensayo me ha servido mucho más adelante de guía para mis proyectos relacionados con la materialidad y la ruina" ${ }^{30}$. Estaba testimoniando con ello dos cuestiones que en este primer libro del arquitecto donostiarra se ponen de manifiesto con particular claridad: la inseparabilidad entre los escritos y los proyectos característica de su obra y, simultáneamente, la profunda continuidad en cuanto a los temas de interés que la recorre y articula. Desde esta perspectiva es posible también comprender mejor cómo, en su planteamiento y en su contenido, aquel precoz trabajo de investigación no puede ser reducido a mero corolario de una teoría más o menos difundida entre los arquitectos españoles en los años setenta, sino que contiene valiosas claves para la posterior trayectoria de su autor
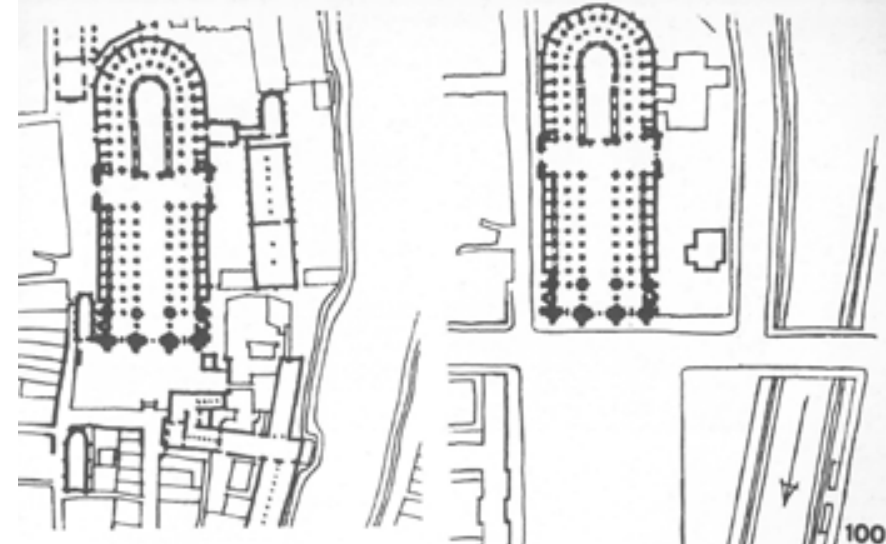
figura 12
Evolucion del entorno de Notre-Dame de
Paris, seguin Linazasoro (Permanencias,
1978 ) París, según Linazasoro (Permanencias,
1978 )
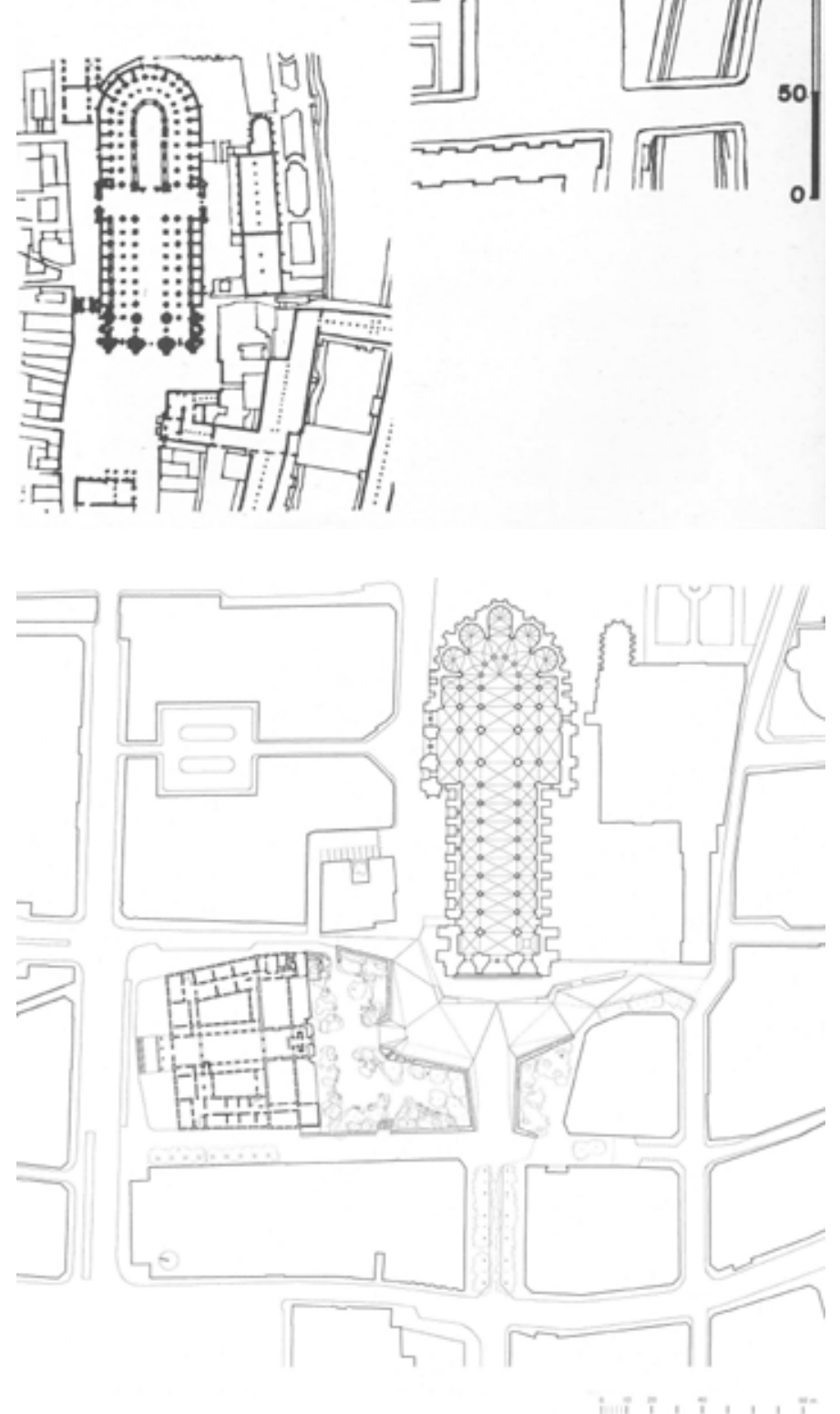
1. GREGOTTI, Vittorio. Nuevos caminos de
la arquitectura italiana. Barcelona: Gustavo 2. 2.
PRIETO, Eduardo. "José Ignacio
Linazasoro, una biografia intelectual".
RITA, ${ }^{\circ} 12,2019$, p. 15.

3. 列 una búsqueda. Sobre escritos y proyectos.
Valladolid: Escuela Técnica Superior de
AArquitectura de Ve Valladolid, 2019, p. 24. El libroc colectivo sobre la ciudad de Padua
fue publicado en 1970 , pero era el resultado fues los trabajos académicos iniciados en la
de Escuela de Venecia durante el curso
66 y coordina-dos por Aymonino.

4. PRIETO, Eduardo. "José Ignacio
Linazasoro, una biografia intelectual".
RITA, no 12, 2019, p. 14 . 5. "Me interesaba la arquitectura románica
hasta el punto de llegar a convencer a mis padres para que me llevaran, por algunas de
las peores carreteras de los años sesenta, a visitar iglesias recónditas que conocia sólo
a traves de fotogra-fias". IINAZASORO, José Ignacio. Memoria de una búsquedi
Sobre escritos $y$ provectos. Valladolide Escuela Tercni-ca Superior
de Valladolid, 2019, p. 20 .

6.

LINAZASORO, José Ignacio. "La critica
del silencio. Giorgio Grassi ylos arquitect hoportunos. En: GRASSI, Giorgio. La arquitectura como oficio y otros es.
Barcelona: Gustavo Gili, 1980, p. 17.

7. GRASSI, Giorgio. La construcción lógica
de la arquitectura. Barcelona: Colegio de la arquitectura. Barcelona: Colegio
Oficial de Arquitectos de Cataluña y
Baleares, 1973, p. 74 . 8. ROSSI, Aldo. LLa arquitectura de la ciudad
Barcelona: Gustavo Gili, 1971 , p.50. 9. Traducción del autor: "Au lieu de nous
initier à la culture lombarde et italienne
it sest efforcé de déveloper Pinterét pour les traditions archirecturales et culturelles suisses. Cést ainsi que les
étudiants ont commencè soccuper des structures des villes et des villages suisses
et à rechercher des liens avec les differentes tradtions "u bati" En: GUBLER, Jacques, ed. Aldo Rossi autobio-graphies partagées. Latuanne:
Presses Polytechniques et Universitaires 10 El capitulo sobre las fuentes bibliograficas de las que el arquitecto explícito al respecto, al remitiri a los trabajo
de Muratori sobre Venecia y Roma, de de Padua, de otro; véase LINAZASORO, José Ignacio. Permanencias y arquitectura urbana. Las ciudades vascas de la época
romana a la llustracíon. Barcelona: Gustavo
Gili 1978
11. GALARRAGA, Iñaki; PEÑA lavorare intorno allition: "Octasione perio "Hirigintza: L.... "La descrizione debe raggiungere
grado di invezione / oltre a descrizion de An-tropologia Vasca, vol. 4. Billaa: L L
Gran Enciclopedia Vasca, 1976, pp. 277. 310. Galarraga se habia incorporado a 12.

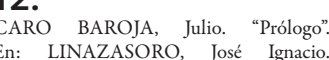
Permanencias y arquitectura urbana. Las
cuidadedes vascas de la epoca romana a la Mudades vascas de la época romana a la
lustración. Barcelona: Gustaro Gili, 1978.

13 Lsa investigación le fue encargada a Ross docencia en el Politécnico de Zür-rich

y aparecería publicada poco después de
la de Linazasoro; véase ROSSI, Aldo;
CONSOLASCO Eas Max. La costruzione del territerio nel
Cantone Ticino. Luganon: Fondazione
Ticino Nostro 1979.

14.

LINAZASORO, José Ignacio Permanencias y arquitectura urbana. Las
ciudades vascas de la eppoca romana a la llustración. Barcelona: Gustavo Gili, 1978.

Tinducción del autor: "Camino e calltc

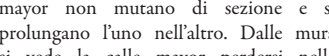
vede la calle mayor perdersi nellic
colline come stradz" ROSSI Aldo. quaderni azzurri, 1968-1992, cuadern maggio 1974, ed. facsimil sin paginat Milan: Electa \& $\&$ The Getty Research
Mutitu-te, 1999 .

16.

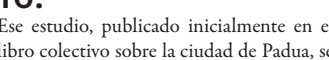
encuentrar aceogido en ROSSI, Aldo. Para una arpuitectura de tendencia. Escritos
1956-1972. 2 arcelona: Gustavo Gili, 1977 .

17.

quadri pacsaggistricið, in Svizzera cos

idea, rica di sviluppi e rissolti operativy

Ortelli del libro de Rossi, Consolascio y
Bosshard, publicada en Urbanisitica n

1986, p.

18.

Iraducción del autor: "Sembra quas
upossibile che la qualitá che la pietra ha

nodo restaurata nel senso di una nuthe

funzionalita delléedificio. [...II I suo destino
si identifica con il sig-nificato storico

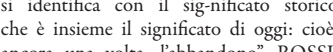

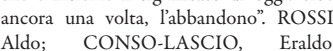

Aldo; CONSO-LASCIO, Eraldo

territorio nel cantone Ticino. Lugano
Fondazione Ticino Nostro, 1979, p. 26 . 16: Architetura. 25 novembre $1977-20$ Permanencias y arguitecerura urbana. Las
ciudades vascas de la epoca romana a Europa. II progetto urbano in Spagna
Napo-li: CLEAN, 2015, p.51. monuments. Bruselas: Mardaga, 1984, p
19.

29.

, José Ignacio. Memoria

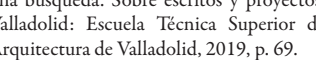

delle carte e dei rilievi". ROSSI, Aldo. maggio 1974, ed. facsimil sin paginat
Milalan: Flecta \& The Getry Research 30.

INAZASORO, José Ignacio. Textos
citicos. Madrid: Asimertricas, 2017, p. 56.

20.

WNAZASORO, José Ignacio. Memoriad

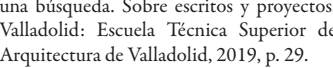

21

Ignacio Sustración. Barcelona: Gustavo Gili, 1978 22.

23.

\section{4.}

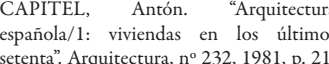

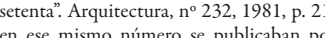

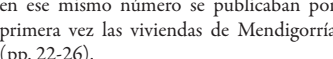

25.

Eduardo. "José Ignacio Linazasoro, una biografia intelectuall
RITA, no 12, 2019, p. 15. Assi lo habis Influenze delly Ton Tho, Francesse.

26.

DEL MONTE, José Mari. "De las virtudes de lo antiguo". En:
LINAZASORO, José Ignacio. Evocando ruina: sombras y texturas. Centro cultural
en Lavapies, Madrid, Madrid: AG Grupo
2004, p. 15.

27.

De hecho, para ilustrarto utiliza una seric relación con la caredral de Notre-Dame de Paris vease LINAZASORO, José Innacio ciudades rascas de la época romana a la
Ilustración. Barcelona: Gustavo Gili, 1978, .174. Recientemente ha vuelto sobre es

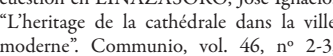
moderne. Communio, vol. $46, \mathrm{n}^{\circ} 2-3$

28.

Una selección de los resultados de ese

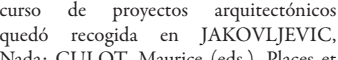

\section{Victoriano Sainz Gutiérrez}

Serita. Doctor arquitecto, 1997. Profesor Titular de la ETSA de Sevilla desde 2000. Profesor visitante del Instituto Superior Técnico de Lisboa Territorio de la Universidad de Sevilla, 2012-2020. Investigerer resión del del grupo de investigación HUM-710 del Plan Andaluz de Investigación desde 2015. Ha publicado, entre otros, los libros: El proyecto urbano en Espań Génesis y desarrollo de un urbanismo de los arquitectos (Sevilla, 2006), Aldo Rossi: la ciudad, la arqutectura, el pensamiento (Buenos Aires, 2011) y Aldo ossi y Sevilla. El significado de unos viajes (Sevilla, 2019). vsainz@us.es

Fuente de financiamiento S.I

\section{Bibliografía}

AA. VV. La città di Padova. Saggio di analisi urbana.

Roma: Officina, 1970.

AmoNNo, Carlo. Origenesy desarrollo de la ciudad moderna. Barcelona: Gustavo Gili, 1973.

\section{CAPITEL, Antón. "Arquitecturas.
Arquitectura, no $232,1981$.}

GALARRAGA, Iñaki; PEÑA GANCHEGUI, Luis. "Hirigintza: construcción de la

En: III Semana de Antropologia Vasca, vol. 4. Bilbao: La Gran Enciclopedia Vasca, 1976.

GARCíA DEL MONTE, José María. "De las virtudes de lo antiguo". En: LINAZASORO, Jose Ignacio. Evocandol la ruina: sombras y texturas.
Centro cultural en Lavapiés, Madrid. Madrid: AG Grupo, 2004, pp. 14-17. GRASSI, Giorgio. La construcción lógica de la arquitectura.

Barcelona: Colegio Oficial de Arquitectos de Cataluña y Baleares, 1973.

GREGOTTI, Vittorio. Nuevos

HELFENSTEIN, Heinrich. "Petit theâtre du souvenir"

En: GUBLER, Jacques, ed. Aldo Rossi: autobiog-raphies partagées. Lausanne: Presses Polytecniques et Universitaires Romandes, 2000.

JAKOVLJEVIC, Nada; CULOT,

LINAZASORO, José Ignacio. Permanencias y arquitectura urbana. Las ciudades vascas de la epoca romana a la llustracion

LINAZASORO, José Ignacio. "La critica del silencio. Giorgio Grassi y los

arquitectosinoportunos".
En: GRASSI, Giorgio. La arquitectura como oficio y otros escritos. Barcelona: Gustavo Gili, 1980

LINAZASORO, José Ignacio. Textos criticos.

Madrid: Asimétricas, 2017

LINAZASORO, José Ignacio. Memoria de una búsqueda. Sobre escritos proyectos.

INAZASORO, José Ignacio. "L'heritage de la cathédrale dans la ville

PRIETO, Eduardo. "José Ignacio Linazasoro, una biografía intelectual"

ROSSI, Aldo. La arquitectura de la ciudad.

Bili, 1971.

ROSSI, Aldo. Para una arquitectura de tendencia. Escritos: 1956-1972.

ROSSI, Aldo. I quaderni azzurri, 1968-1992,

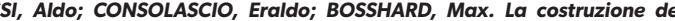

SORRENTINO, Francesco. Influenze della Tendenza italiana in Europa. II

progetto urbano in Spagna.
Napoli: CLEAN, 2015. 